hep-th/0504033

$\mathrm{IC} / 2005 / 13$

\title{
A New Anomaly-Free Gauged Supergravity in Six Dimensions
}

\author{
Spyros D. Avrami-* and Alex Kehagia: \\ Department of Physics, National Technical University of Athens, GR-15773, Zografou, Athens, Greece \\ S. Randjbar-Daem \\ International Center for Theoretical Physics, 34100, Trieste, Italy
}

(Dated: November 6, 2018)

\begin{abstract}
We present a new anomaly-free gauged $\mathcal{N}=1$ supergravity model in six dimensions. The gauge group is $E_{7} \times G_{2} \times U(1)_{R}$, with all hyperinos transforming in the product representation $(\mathbf{5 6}, \mathbf{1 4})$. The theory admits monopole compactifications to $\mathbb{R}^{4} \times \mathbf{S}^{2}$, leading to $D=4$ effective theories with broken supersymmetry and massless fermions.
\end{abstract}

\section{INTRODUCTION}

Minimal supergravity theories in six dimensions have a remarkably rich structure and have attracted much interest over the years. Some of the reasons motivating the study of such theories are their connection to superstring vacua, their relation to $\mathcal{N}=2$ theories in $D=4$ and the framework they provide for cosmological investigations. Among the most interesting models of this type are $D=6$ gauged supergravity models which have the important property that they spontaneously compactify on lower-dimensional spaces. A prototype for such compactifications is employed in the Salam-Sezgin model 1], a $D=6$ supersymmetric Einstein-Maxwell theory. This theory admits an $\mathbb{R}^{4} \times \mathbf{S}^{2}$ solution that preserves half the supersymmetries, obtained through a magnetic monopole background residing on $\mathbf{S}^{2}$ and, as has recently been shown [2], it is the unique maximally-symmetric solution in this class of models.

One further interesting aspect of the monopole compactification in the supersymmetric models is the possibility of making all the $U(1)$ factors in the gauge group massive. In the non-supersymmetric Einstein-Maxwell theory in six dimensions, the monopole compactification gives rise to an effective $D=4$ chiral gauge theory with gauge group $S U(2)_{K K} \times U(1)$. The chirality of the $D=4$ effective theory is due to the $U(1)$ factor which remains massless and has complex representations. In the supersymmetric generalizations, such as the Salam-Sezgin model or the non-Abelian theories discussed in this pa-

\footnotetext{
*Electronic address: avramis@cern.ch

${ }^{\dagger}$ Electronic address: kehagias@cern.ch

${ }^{\ddagger}$ Electronic address: seif@ictp.trieste.it
}

per, the vector potentials associated to the $U(1)$ factors of magnetic monopoles acquire a mass due to their ChernSimons coupling to the second-rank antisymmetric potentials. This coupling is an essential ingredient of all such models.

In addition to the nonzero mass for the $U(1)$ gauge potentials in the monopole directions, most $D=6 \mathrm{su}-$ pergravity theories, including the Salam-Sezgin model, suffer from the breakdown of local symmetries due to the presence of gravitational, gauge and mixed anomalies [3] which render such theories inconsistent at the quantum level. In fact, anomaly cancellation has turned out to be a crucial guiding principle for the identification of consistent $D=6$ theories for the same reason as in the $D=10$ case. Although the $D=6$ anomaly cancellation conditions are weaker than those in $D=10$, they are still very stringent, especially in the case of gauged supergravity theories.

Regarding Poincaré supergravities, there are numerous anomaly-free theories in the literature. Most of these were found by compactifying heterotic string theory on $K 3$ using various methods [4] of embedding the $K 3$ holonomy group in the $S O(32)$ or $E_{8} \times E_{8}$ gauge group. There are also theories with enhanced symmetry originating from the Gepner points of orbifold realizations of $K 3$ [5] or from the non-perturbative mechanism of small instantons [6], as well as theories found by solving the anomaly cancellation conditions alone 7]. Such theories can also be constructed as boundary theories on the six-dimensional orbifold fixed points in seven dimensions compactified on $\mathbf{S}^{1} / \mathbb{Z}_{2}$ [8, 9].

However, in the case of gauged supergravities, there is only one known non-trivial anomaly-free model, namely the $E_{7} \times E_{6} \times U(1)_{R}$ model of [10]. This model contains 456 hypermatter fields, identified as a halfhypermultiplet in the pseudoreal 912 of $E_{7}$. The theory 
satisfies a set of highly non-trivial anomaly constraints which make it possible to completely cancel all anomalies by the Green-Schwarz mechanism. Moreover, gauging of $U(1)_{R}$ gives rise to a positive-definite potential which implies that one may turn on a magnetic monopole background in a $U(1)$ subgroup of the gauge group and compactify the theory on $\mathbb{R}^{4} \times \mathbf{S}^{2}$. In the particular case considered in [10], where the monopole is embedded in the "hidden" $E_{6}$, the fermionic zero modes come exclusively from the $E_{6}$ gauginos. The resulting $D=4$ theory has an $S O(10) \times U(1)_{R}$ gauge symmetry. Unlike the SalamSezgin model, supersymmetry is completely broken. For the minimal value of the monopole number, which is required by the stability of the compactification, one obtains two chiral families of $S O(10)$ in the 16-dimensional spinor representation.

One very attractive property of the minimal gauged supergravities in $D=6$ is that, unlike the superstring theories in $D=10$, they do not admit the flat spaces as their most symmetric solutions. On the other hand the $\mathbb{R}^{4} \times \mathbf{S}^{2}$ configuration is the unique maximallysymmetric compactification [2]. Furthermore, the expectation values of all the scalars in the model, with the exception of the dilaton, are uniquely determined at the tree level. Hence there is only one modulus accompanying these vacua. Like the $D=10$ supergravities, these models also admit brane solutions of various dimensions 2, 13, 14, 16, 17. Because of the uniqueness and simplicity of the $\mathbb{R}^{4} \times \mathbf{S}^{2}$ compactification as well as many shared features with the $D=10$ heterotic theory it is useful to construct more models of this type and study their low-energy physics.

In this paper, we demonstrate the existence of a new gauged anomaly-free $D=6, \mathcal{N}=1$ model, besides the known one of [10]. The gauge group here is $E_{7} \times G_{2} \times U(1)_{R}$ and so the theory contains 148 vector multiplets. Restricting to onnly one tensor multiplet, cancellation of the irreducible gravitational anomaly demands then 392 hypermultiplets. These fit exactly into a half-hypermultiplet of the pseudoreal product representation $(\mathbf{5 6}, \mathbf{1 4})$ of $E_{7} \times G_{2}$. Again, it is remarkable that all anomalies of the theory cancel in a non-trivial way. Moreover, the model is also free of global anomalies 18, 19] that could potentially arise due to the presence of the $G_{2}$ factor. Regarding the bosonic sector, the 1568 real hyperscalars parameterize the quaternionic manifold $S p(392,1) / S p(392) \times S p(1)$ and gauging of $U(1)_{R}$ contained in $S p(1)$ and $E_{7} \times G_{2}$ contained in $S p(392)$ yields a positive-definite potential allowing $\mathbb{R}^{4} \times \mathbf{S}^{2}$ compactifications. Unlike the $E_{7} \times E_{6} \times U(1)_{R}$ case, there are fermionic zero modes coming from both the gauginos of the $E_{7} \times G_{2}$ subgroup where the monopole is embedded and the hyperinos, since the latter transform non-trivially under both $E_{7}$ and $G_{2}$ factors. These compactifications generate a rich spectrum of chiral fermions in the 27's (or 16's ) of the unbroken $E_{6}$ (or $S O(10)$ ). However, as we shall show, they are perturbatively unstable. To find an anomaly-free model with a realistic $D=4$ fermion spectrum still remains a challenging and unsolved problem.

This paper is organized as follows. In Section [I] we fix our notation, we describe the basic aspects of gauged $D=6$ supergravity theories and we write the bosonic Lagrangian of our model. In Section [II] we explicitly show that the theory is free of anomalies. In Section IV we discuss compactification of the theory on $\mathbb{R}^{4} \times \mathbf{S}^{2}$ and we briefly consider various aspects of the effective $D=4$ theory. In Section $\nabla$ we discuss the spectrum of $D=4$ chiral fermions in detail and show the existence of the tachyonic mode in the spectrum of most compactifications. Finally, in Section [V] we summarize and conclude.

\section{THE MODEL}

The building blocks of $D=6, \mathcal{N}=1$ supergravity theories are the massless representations of the minimal supersymmetry algebra which is chiral and has $S p(1)$ as its R-symmetry group. The field content of these representations is summarized in the following multiplets

$$
\begin{array}{rll}
\text { Supergravity multiplet } & : & \left(g_{M N}, B_{M N}^{-}, \psi_{M}^{i-}\right), \\
\text { Tensor multiplet } & : & \left(B_{M N}^{+}, \phi, \chi^{i+}\right), \\
\text { Vector multiplet } & : & \left(A_{M}, \lambda^{i-}\right), \\
\text { Hypermultiplet } & : & \left(4 \varphi, 2 \psi^{+}\right),
\end{array}
$$

where, the $+(-)$ superscripts denote positive (negative) chirality for the spinors and (anti-)self-duality for 2 -forms and the index $i=1,2$ takes values in the fundamental of $S p(1)_{R}$.

A general $D=6, \mathcal{N}=1$ supergravity theory coupled to matter is constructed by combining one supergravity multiplet with $n_{T}$ tensor multiplets, $n_{V}$ vector multiplets and $n_{H}$ hypermultiplets. Generic string and M-theory compactifications may produce all of these multiplets with arbitrary values of $n_{T}$. Anomaly cancellation using the Green-Schwarz mechanism, however, restricts these numbers by the constraint 10]

$$
n_{H}=n_{V}+273-29 n_{T} .
$$

Starting from the tensor multiplets, we will restrict to the case $n_{T}=1$, where there exists a covariant Lagrangian description of the model. For this case, the constraint given above reduces to

$$
n_{H}=n_{V}+244 .
$$


Regarding the hypermultiplets, the $4 n_{H}$ hyperscalars must parameterize a non-compact quaternionic manifold, whose possible forms are given in [11]; here, we will consider the case where the hyperscalar manifold is $S p\left(n_{H}, 1\right) / S p\left(n_{H}\right) \times S p(1)_{R}$. Finally, the vector multiplets must belong to a gauge group that is a subgroup of the $S p\left(n_{H}, 1\right)$ isometry group of the quaternionic manifold, possibly times extra factors under which the hypermultiplets transform as singlets (the $E_{6}$ in $[10$ is one such example). Aside from such factors, one usually chooses the gauge group to be a product of a subgroup of $S p\left(n_{H}\right)$ and a subgroup of $S p(1)_{R}$. Under the first factor, the hyperinos may transform in arbitrary representations while the gravitino and tensorino are inert. Under the second factor, the hyperinos are inert (although the hyperscalars are charged) while the gravitino, tensorino and gauginos transform non-trivially. Here, we will consider a $U(1)_{R}$ subgroup in which case the gravitino, the tensorino and the gauginos as well as the hyperscalars have all unit charge.

In our model, we pick the gauge group to be $E_{7} \times G_{2} \times$ $U(1)_{R}$. Thus, we have a total of $133+14+1=148$ vector multiplets and Eq. (3) requires that the total number of hypermultiplets be equal to $148+244=392$. The hyperinos fit nicely (no singlets!) into a half-hypermultiplet of the pseudoreal 784-dimensional representation $(\mathbf{5 6}, \mathbf{1 4})$. So, the transformation properties of the various fermions under the three gauge group factors are as follows

$$
\begin{aligned}
\psi_{M}^{-}: & (\mathbf{1}, \mathbf{1})_{1} \\
\chi^{+}: & (\mathbf{1}, \mathbf{1})_{1} \\
\lambda^{-}: & (\mathbf{1 3 3}, \mathbf{1})_{1}+(\mathbf{1}, \mathbf{1 4})_{1}+(\mathbf{1}, \mathbf{1})_{1} \\
\psi^{+}: & \frac{1}{2}(\mathbf{5 6}, \mathbf{1 4})_{0}
\end{aligned}
$$

where the subscripts indicate $U(1)_{R}$ charges.

Passing on to the hyperscalars, they parameterize the manifold

$$
\mathcal{M}=\frac{S p(392,1)}{S p(392) \times S p(1)_{R}},
$$

where the holonomy group in the denominator corresponds to the (unbroken) local symmetry of the scalar sector. One may then gauge a subgroup of the isometry group $S p(392,1)$; in our case we will consider the gauging of $E_{7} \times G_{2} \times U(1)_{R}$. The embedding of $E_{7} \times G_{2}$ in $S p(392)$ is defined by identifying $(\mathbf{5 6}, \mathbf{1 4})$ with the pseudoreal fundamental representation $\mathbf{7 8 4}$ of $S p(392)$.

The construction of the gauged supergravity theory proceeds along the steps described in 11, 12]; for the $E_{7} \times E_{6} \times U(1)_{R}$ case, this procedure was outlined in 10] and discussed in great detail in 16 and can be applied with minor modifications to our model as well. The only aspects of this construction that need attention refer to the hyperscalar sector. To begin, we let $\alpha=1, \ldots, 4 \times 392$ label the coordinates on $\mathcal{M}$ and $a=1, \ldots, 2 \times 392$ label the fundamental of $S p(392)$. We then pick a gauge-fixed coset representative $L$ and we decompose its MaurerCartan form into the coset vielbein and the $S p(392)$ and $S p(1)_{R}$ connections

$$
\begin{aligned}
V_{\alpha}^{a i} & =\left(L^{-1} \partial_{\alpha} L\right)^{a i}, \\
\mathcal{A}_{\alpha}^{a b} & =\left(L^{-1} \partial_{\alpha} L\right)^{a b}, \\
\mathcal{A}_{\alpha}^{i j} & =\left(L^{-1} \partial_{\alpha} L\right)^{i j},
\end{aligned}
$$

whose pullbacks on the spacetime manifold define the composite vielbein and connections

$$
\begin{aligned}
P_{M}^{a i} & =\left(L^{-1} \partial_{M} L\right)^{a i}=\partial_{M} \varphi^{\alpha} V_{\alpha}^{a i}, \\
Q_{M}^{a b} & =\left(L^{-1} \partial_{M} L\right)^{a b}=\partial_{M} \varphi^{\alpha} \mathcal{A}_{\alpha}^{a b}, \\
Q_{M}^{i j} & =\left(L^{-1} \partial_{M} L\right)^{i j}=\partial_{M} \varphi^{\alpha} \mathcal{A}_{\alpha}^{i j},
\end{aligned}
$$

that are used to construct scalar kinetic terms and spinor covariant derivatives respectively. Gauging $E_{7} \times G_{2} \times$ $U(1)_{R}$ entails introducing the $E_{7}$ gauge fields $A_{M}^{I}, I=$ $1, \ldots, 133$, the $G_{2}$ gauge fields $A_{M}^{I^{\prime}}, I^{\prime}=1, \ldots, 14$ and the $U(1)_{R}$ gauge field $A_{M}^{3}$ and replacing ordinary derivatives by gauge-covariant ones. In particular, the covariant derivative acting on the hyperscalars is

$$
\mathcal{D}_{M} \varphi^{\alpha}=\partial_{M} \varphi^{\alpha}-g\left(A_{M}^{I} \xi^{I \alpha}+A_{M}^{I^{\prime}} \xi^{I^{\prime} \alpha}\right)-g^{\prime} A_{M}^{3} \xi^{3 \alpha},
$$

where $T^{I}, T^{I^{\prime}}$ and $T^{3}$ are the antihermitian $E_{7}, G_{2}$ and $U(1)_{R}$ generators, $\xi^{I \alpha}=\left(T^{I} \varphi\right)^{\alpha}, \xi^{I^{\prime} \alpha}=\left(T^{I^{\prime}} \varphi\right)^{\alpha}$ and $\xi^{3 \alpha}=\left(T^{3} \varphi\right)^{\alpha}$ are Killing vectors associated with the respective isometries and $g$ and $g^{\prime}$ are the $E_{7} \times G_{2}$ and $U(1)_{R}$ couplings. Accordingly, the composite vielbein and connections in (7) are replaced by the gauged versions

$$
\begin{aligned}
& \mathcal{P}_{M}^{a i}=\left(L^{-1} \mathcal{D}_{M} L\right)^{a i}=\mathcal{D}_{M} \varphi^{\alpha} V_{\alpha}^{a i}, \\
& \mathcal{Q}_{M}^{a b}=\left(L^{-1} \mathcal{D}_{M} L\right)^{a b}=\mathcal{D}_{M} \varphi^{\alpha} \mathcal{A}_{\alpha}^{a b}, \\
& \mathcal{Q}_{M}^{i j}=\left(L^{-1} \mathcal{D}_{M} L\right)^{i j}=\mathcal{D}_{M} \varphi^{\alpha} \mathcal{A}_{\alpha}^{i j}-g^{\prime} A_{M}^{3}\left(T^{3}\right)^{i j}
\end{aligned}
$$

A direct consequence of the gauging of $U(1)_{R}$ is the emergence of a scalar potential in the theory. This comes about due to the fact that the commutator $\left[\mathcal{D}_{M}, \mathcal{D}_{N}\right] \epsilon^{i}$ appearing in the supersymmetry variation of the gravitino kinetic term acquires an extra term involving the gauge field strengths and the functions

$$
\begin{gathered}
C_{i j}^{I}=\left(L^{-1} T^{I} L\right)_{i j}, \quad C_{i j}^{I^{\prime}}=\left(L^{-1} T^{I^{\prime}} L\right)_{i j}, \\
C_{i j}^{3}=\left(L^{-1} T^{3} L\right)_{i j} .
\end{gathered}
$$

Restoring local supersymmetry requires then a set of modifications to the Lagrangian and transformation rules; in the bosonic sector, this induces a hyperscalar potential. 
The Lagrangian of the gauged supergravity theory was first derived in [1] and further elaborated upon in [12]. Its bosonic part may be written as

$$
\begin{aligned}
e^{-1} \mathcal{L}= & \frac{1}{4} R-\frac{1}{4} \partial_{M} \phi \partial^{M} \phi-\frac{1}{12} e^{2 \phi} G_{M N P} G^{M N P} \\
& -\frac{1}{4} e^{\phi} v_{A} \operatorname{tr}\left(F_{A M N} F_{A}^{M N}\right) \\
& -g_{\alpha \beta}(\varphi) \mathcal{D}_{M} \varphi^{\alpha} \mathcal{D}^{M} \varphi^{\beta} \\
& +\frac{1}{8} e^{-1} \epsilon^{M N P Q R S} B_{M N} v_{A} \operatorname{tr}\left(F_{A P Q} F_{A R S}\right) \\
& -\frac{1}{4} e^{-\phi}\left[g^{2}\left(v_{7}^{-1} C_{i j}^{I} C^{I i j}+v_{2}^{-1} C_{i j}^{I^{\prime}} C^{I^{\prime} i j}\right)\right. \\
& \left.+g^{\prime 2} v_{1}^{-1} C_{i j}^{3} C^{3 i j}\right] .
\end{aligned}
$$

Here, the summation index $A=7,2,1$ runs over the three gauge group factors, $v_{\alpha}$ are a set of constants to be determined later and the various traces are interpreted as e.g. $\operatorname{tr}\left(F_{7 M N} F_{7}^{M N}\right)=F_{M N}^{I} F^{I M N}$. In this form of the Lagrangian, the field strength of $B_{M N}$ is given by the usual definition $G_{M N P}=3 \partial_{[M} B_{N P]}$ and the Green-Schwarz term is explicit.

\section{ANOMALY CANCELLATION}

In this section, we demonstrate that the theory described above is anomaly-free. Generally, $D=6$ chiral supergravities suffer from gravitational, gauge and mixed anomalies arising from box diagrams with four external gravitons and/or gauge bosons and one chiral spinor or (anti-)self-dual 2-form running in the loop. Starting from the gravitational anomalies, we first note that the contributions from the 2 -forms from the supergravity and tensor multiplets cancel each other so that the only nonzero terms come from the gravitino, tensorino, gauginos and hyperinos. Summing these contributions (in that order) using the formulas of the Appendix, we find the expression

$$
\begin{aligned}
I_{8}(R)= & -\left[\frac{49}{72} \operatorname{tr} R^{4}-\frac{43}{288}\left(\operatorname{tr} R^{2}\right)^{2}\right] \\
& +(1-148+392)\left[\frac{1}{360} \operatorname{tr} R^{4}+\frac{1}{288}\left(\operatorname{tr} R^{2}\right)^{2}\right] \\
= & \left(\operatorname{tr} R^{2}\right)^{2}
\end{aligned}
$$

which confirms that the irreducible tr $R^{4}$ terms cancel and explains the particular normalization chosen. Turning to the gauge anomalies, these may be split into (i) $E_{7}$ and $G_{2}$ anomalies (contributions from gauginos and hyperinos), (ii) $U(1)_{R}$ anomalies (contributions from gravitino, tensorino and gauginos), (iii) $E_{7} \times G_{2}$ anomalies (contributions from hyperinos) and (iv) $E_{7} \times U(1)_{R}$ and $G_{2} \times U(1)_{R}$ anomalies (contributions from gauginos).
Writing down the various contributions in the order indicated above, we find

$$
\begin{aligned}
I_{8}(F)= & \frac{2}{3}\left(-\operatorname{Tr} F_{7}^{4}+7 \operatorname{tr} F_{7}^{4}\right)+\frac{2}{3}\left(-\operatorname{Tr} F_{2}^{4}+28 \operatorname{Tr} F_{2}^{4}\right) \\
& +\frac{2}{3}(-5+1-148) F_{1}^{4} \\
& +2 \operatorname{tr} F_{7}^{2} \operatorname{Tr} F_{2}^{2} \\
& -4 \operatorname{Tr} F_{7}^{2} F_{1}^{2}-4 \operatorname{Tr} F_{2}^{2} F_{1}^{2} \\
= & -\frac{2}{3} \operatorname{Tr} F_{7}^{4}+\frac{14}{3} \operatorname{tr} F_{7}^{4}+18 \operatorname{Tr} F_{2}^{4}-\frac{304}{3} F_{1}^{4} \\
& +2 \operatorname{tr} F_{7}^{2} \operatorname{Tr} F_{2}^{2}-4 \operatorname{Tr} F_{7}^{2} F_{1}^{2}-4 \operatorname{Tr} F_{2}^{2} F_{1}^{2} . \quad(13)
\end{aligned}
$$

where "tr" and "Tr" stand for fundamental and adjoint traces respectively. Finally, we have to consider the mixed anomalies. Splitting them into (i) mixed $E_{7}$ and $G_{2}$ anomalies (contributions from gauginos and hyperinos) and (ii) mixed $U(1)_{R}$ anomalies (contributions from gravitino, tensorino and gauginos), we write

$$
\begin{aligned}
I_{8}(F, R)= & \frac{1}{6} \operatorname{tr} R^{2}\left(\operatorname{Tr} F_{7}^{2}-7 \operatorname{tr} F_{7}^{2}+\operatorname{Tr} F_{2}^{2}-28 \operatorname{Tr} F_{2}^{2}\right) \\
& +\frac{1}{6}(-19-1+148) \operatorname{tr} R^{2} F_{1}^{2} \\
= & \operatorname{tr} R^{2}\left(\frac{1}{6} \operatorname{Tr} F_{7}^{2}-\frac{7}{6} \operatorname{tr} F_{7}^{2}-\frac{9}{2} \operatorname{Tr} F_{2}^{2}\right) \\
& +\frac{64}{3} \operatorname{tr} R^{2} F_{1}^{2} .
\end{aligned}
$$

Eqs. (13 14 can be simplified by expressing all traces in the fundamental representations. Moreover, since both $E_{7}$ and $G_{2}$ factors do not possess fourth-order invariants, all of the above traces can be expressed exclusively in terms of second-order traces. For the two factors, we have the identities

$$
\begin{array}{cl}
\operatorname{Tr} F_{7}^{2}=3 \operatorname{tr} F_{7}^{2}, & \operatorname{Tr} F_{2}^{2}=4 \operatorname{tr} F_{2}^{2}, \\
\operatorname{tr} F_{7}^{4}=\frac{1}{24}\left(\operatorname{tr} F_{7}^{2}\right)^{2}, & \operatorname{tr} F_{2}^{4}=\frac{1}{4}\left(\operatorname{tr} F_{2}^{2}\right)^{2}, \\
\operatorname{Tr} F_{7}^{4}=\frac{1}{6}\left(\operatorname{tr} F_{7}^{2}\right)^{2}, & \operatorname{Tr} F_{2}^{4}=\frac{5}{2}\left(\operatorname{tr} F_{2}^{2}\right)^{2} .
\end{array}
$$

Substituting these in (13) and (14), we write the gauge and mixed anomalies in the simplified form

$$
\begin{aligned}
I_{8}(F)= & \frac{1}{12}\left(\operatorname{tr} F_{7}^{2}\right)^{2}+45\left(\operatorname{tr} F_{2}^{2}\right)^{2}-\frac{304}{3} F_{1}^{4} \\
& +8 \operatorname{tr} F_{7}^{2} \operatorname{tr} F_{2}^{2}-12 \operatorname{tr} F_{7}^{2} F_{1}^{2}-16 \operatorname{tr} F_{2}^{2} F_{1}^{2},
\end{aligned}
$$

and

$$
I_{8}(F, R)=\operatorname{tr} R^{2}\left(-\frac{2}{3} \operatorname{tr} F_{7}^{2}-18 \operatorname{tr} F_{2}^{2}+\frac{64}{3} F_{1}^{2}\right) .
$$

Putting the three contributions (12), (16) and (17) together, we find that the total anomaly polynomial is given by

$$
I_{8}=\left(\operatorname{tr} R^{2}\right)^{2}
$$




$$
\begin{aligned}
& +\operatorname{tr} R^{2}\left(-\frac{2}{3} \operatorname{tr} F_{7}^{2}-18 \operatorname{tr} F_{2}^{2}+\frac{64}{3} F_{1}^{2}\right) \\
& +\frac{1}{12}\left(\operatorname{tr} F_{7}^{2}\right)^{2}+45\left(\operatorname{tr} F_{2}^{2}\right)^{2}-\frac{304}{3} F_{1}^{4} \\
& +8 \operatorname{tr} F_{7}^{2} \operatorname{tr} F_{2}^{2}-12 \operatorname{tr} F_{7}^{2} F_{1}^{2}-16 \operatorname{tr} F_{2}^{2} F_{1}^{2} .
\end{aligned}
$$

In order for the Green-Schwarz mechanism to operate, the above polynomial must factorize as

$$
I_{8}=\left(\operatorname{tr} R^{2}+u_{A} \operatorname{tr} F_{A}^{2}\right)\left(\operatorname{tr} R^{2}+\tilde{u}_{A} \operatorname{tr} F_{A}^{2}\right) .
$$

To check whether this is possible, one must (i) compare the $\left(\operatorname{tr} F_{A}^{2}\right)^{2}$ and $\operatorname{tr} R^{2} \operatorname{tr} F_{A}^{2}$ terms to determine $\left(u_{A}, \tilde{u}_{A}\right)$ and (ii) check if, for the values of $\left(u_{A}, \tilde{u}_{A}\right)$ thus determined, the $\operatorname{tr} F_{A}^{2} \operatorname{tr} F_{B}^{2}$ cross-terms match as well. Remarkably, it turns out that all conditions are indeed satisfied with

$$
\begin{array}{ll}
u_{7}=-\frac{1}{2}, & u_{2}=-3, \quad u_{1}=-4 \\
\tilde{u}_{7}=-\frac{1}{6}, & \tilde{u}_{2}=-15, \quad \tilde{u}_{1}=\frac{76}{3} .
\end{array}
$$

Cancellation of anomalies is then a straightforward matter. One may set the undetermined constants $v_{A}$ in (11) equal to

$$
v_{A}=-u_{A},
$$

and modify the $B_{M N}$ gauge transformation law to

$$
\delta B_{2} \sim \omega_{2 L}^{1}+\tilde{u}_{A} \omega_{2 Y, A}^{1},
$$

where $\omega_{2 L}^{1}$ and $\omega_{2 Y, A}^{1}$ are related to $\operatorname{tr} R^{2}$ and $\operatorname{tr} F_{A}^{2}$ by descent. The corresponding anomalous variation of the Green-Schwarz term in (11) (including a gravitational term) cancels exactly the variation of the effective action.

Apart from the perturbative anomalies discussed above, our model may also have global anomalies. In particular, since the $G_{2}$ factor in the gauge group has a non-trivial sixth homotopy group, $\pi_{6}\left(G_{2}\right)=\mathbb{Z}_{3}$, there exist gauge transformations not continuously connected to the identity. Under such transformations, the effective action may pick up a phase factor and is thus ill-defined. The condition for the absence of global anomalies in the case of the $G_{2}$ gauge group is given by [20]

$$
1-4 \sum_{\mathcal{R}} n_{\mathcal{R}} b_{\mathcal{R}}=0 \bmod 3,
$$

where $n_{\mathcal{R}}$ is the number of hypermultiplets transforming in the representation $\mathcal{R}$ of $G_{2}$ and $b_{\mathcal{R}}$ is defined by $\operatorname{tr}_{\mathcal{R}} F_{2}^{4}=b_{\mathcal{R}}\left(\operatorname{tr} F_{2}^{2}\right)^{2}$. In our model, the hypermultiplets are in the adjoint of $G_{2}$ and we have $n_{\mathbf{1 4}}=\frac{1}{2} \times 56=28$ and $b_{\mathbf{1 4}}=\frac{5}{2}$. For these numbers, the condition (23) is indeed satisfied and thus the theory is free of global anomalies as well.

\section{COMPACTIFICATION}

The bosonic Lagrangian (11) contains a hyperscalar potential given by its last term. Employing the definitions $g_{7}=g / \sqrt{v_{7}}, g_{2}=g / \sqrt{v_{2}}$ and $g_{1}=g^{\prime} / \sqrt{v_{1}}$, we rewrite this potential as

$$
V(\varphi)=\frac{1}{4} e^{-\phi}\left(g_{7}^{2} C_{i j}^{I} C^{I i j}+g_{2}^{2} C_{i j}^{I^{\prime}} C^{I^{\prime} i j}+g_{1}^{2} C_{i j}^{3} C^{3 i j}\right),
$$

where the various $C$-functions are given in (10). In 16], it was shown that a convenient parameterization of the scalar coset is given by the $784 \times 2$ matrix

$$
\varphi=\left(\begin{array}{c}
\varphi_{1} \\
\vdots \\
\varphi_{392}
\end{array}\right)
$$

where $\varphi_{n}, n=1, \ldots, 392$, are themselves $2 \times 2$ matrices satisfying the reality condition $\varphi_{n}^{*}=\sigma_{2} \varphi_{n} \sigma_{2}$. With this parameterization, one may define the coset representative as the $(784+2) \times(784+2)$ matrix

$$
L=\left(\begin{array}{cc}
1+\left(\frac{\sqrt{1+\varphi^{\dagger} \varphi}-1}{\varphi^{\dagger} \varphi}\right) \varphi \varphi^{\dagger} & \varphi \\
\varphi^{\dagger} & \sqrt{1+\varphi^{\dagger} \varphi}
\end{array}\right),
$$

where the factor inside parentheses is understood as a scalar (since $\varphi^{\dagger} \varphi$ is proportional to the identity). Using the definition (26), it can be shown that the $C$-functions take the form

$$
\begin{gathered}
C_{i j}^{I}=\left(\varphi^{\dagger} T^{I} \varphi\right)_{i j}, \quad C_{i j}^{I^{\prime}}=\left(\varphi^{\dagger} T^{I^{\prime}} \varphi\right)_{i j}, \\
C_{i j}^{3}=\left[1+\operatorname{tr}\left(\varphi^{\dagger} \varphi\right)\right]\left(T^{3}\right)_{i j} .
\end{gathered}
$$

Then the potential is given by the simple expression

$$
\begin{aligned}
V(\varphi)= & \frac{1}{16} e^{-\phi}\left[-g_{7}^{2}\left(\varphi^{\dagger} T^{I} \varphi\right)^{2}-g_{2}^{2}\left(\varphi^{\dagger} T^{I^{\prime}} \varphi\right)^{2}\right] \\
& +\frac{1}{8} e^{-\phi} g_{1}^{2}\left[1+\operatorname{tr}\left(\varphi^{\dagger} \varphi\right)\right]^{2} .
\end{aligned}
$$

Using this expression and recalling that $T^{I}$ and $T^{I^{\prime}}$ are antihermitian, we immediately see that the potential is strictly positive-definite and attains its unique global minimum at $\varphi^{\alpha}=0$. Among other things, this implies that the $E_{7} \times G_{2}$ gauge symmetry cannot be spontaneously broken by the hyperscalars at tree level.

At $\varphi^{\alpha}=0$, the potential (28) takes the exponential form

$$
V_{\min }=\frac{1}{8} e^{-\phi} g_{1}^{2},
$$

and, for the case of constant $\phi$, it acts like a cosmological constant. It is this effective cosmological constant and the particular form of the dilaton coupling which picks 
up the $\mathbb{R}^{4} \times \mathbf{S}^{2}$ among other maximally-symmetric spaces 1]. In the non-supersymmetric theory, de Sitter or antide Sitter spaces would also be possible solutions [15].

In the $E_{7} \times E_{6} \times U(1)_{R}$ model of [10], the monopole was embedded in the $E_{6}$ factor, yielding a $D=4$ effective theory with $S O(10) \times U(1)_{R}$ gauge symmetry where all massless fermions originate from the $E_{6}$ gauginos since only the latter couple to the monopole; in 16] this was generalized to include monopole embeddings in $E_{7}$ where zero-mode fermions arise from the hyperinos as well. In the model considered here, one can embed as many magnetic monopoles as the rank of the gauge group which is 10. In general we will thus have a maximum of 10 monopole charges. This will give rise to fermionic zero modes from the associated gauginos but, since the hyperinos are charged under both $E_{7}$ and $G_{2}$ gauge group factors, it will also necessarily give rise to fermionic zero modes from the associated hyperinos. In the absence of a vev for the vector potential associated to $U(1)_{R}$, the gravitino, tensorino and the rest of the gauginos will be massive. Turning to the bosons, the squared mass of each one of the lightest hyperscalar fluctuations will receive two contributions, one being proportional to the associated eigenvalue of $\frac{\partial^{2} V}{\partial \varphi^{\alpha} \partial \varphi^{\beta}}$ at $\varphi^{\alpha}=0$ and the other being proportional to $D^{2}$ where $D$ is the covariant derivative acting on the hyperscalar fluctuations in the background of the monopole vector potential(s). The first contribution will make all hyperscalars massive. The second contribution, if the monopole charges do not add up to zero, will be a positive quantity proportional to $1 / a^{2}$, where $a$ is the radius of $\mathbf{S}^{2}$. Furthermore in the case of a nonzero net monopole charge of the hyperscalar even the leading (lightest) $D=4$ scalar modes resulting from it will belong to a non trivial irreducible representation of the Kaluza-Klein $S U(2)$. We shall comment on the masses of some other bosonic modes in Section VI]

In the absence of a vev for the $U(1)_{R}$ gauge field, the supersymmetric variation of the gravitino will be nonzero and thus this class of compactifications will break all supersymmetries.

To write down the ansatz for monopole compactification, we employ the rescalings $A_{M}^{I} \rightarrow A_{M}^{I} / g, A_{M}^{I^{\prime}} \rightarrow$ $A_{M}^{I^{\prime}} / g$ and $A_{M}^{3} \rightarrow A_{M}^{3} / g^{\prime}$ and we set the metric, the $U(1) \subset E_{7}$ gauge field and the dilaton equal to

$$
\begin{gathered}
d s_{6}^{2}=\eta_{\mu \nu} d x^{\mu} d x^{\nu}+a^{2}\left(d \theta^{2}+\sin ^{2} \theta d \varphi^{2}\right), \\
A_{ \pm}=\frac{n}{2} Q(\cos \theta \mp 1) d \varphi ; \quad F=\frac{n}{2} Q \sin \theta d \theta \wedge d \varphi, \\
\phi=\phi_{0}=\text { const. }
\end{gathered}
$$

Here, $A_{+}$and $A_{-}$correspond to the potentials on the northern and southern hemisphere which, on the equator, should be connected by a gauge transformation parameterized by $U=e^{i n Q \varphi}$. In order for $U$ to be single-valued as $\varphi$ changes by $2 \pi$, the quantity $n q_{\min }$, where $q_{\min }$ is the minimal $U(1)_{M}$ charge in the theory, must be an integer. In the above, $Q$ is any generator of the gauge group and, in general, can be a linear combination of all commuting generators with appropriate quantization conditions on the coefficients. This ansatz solves the field equations [10].

To prepare the setting for the spectrum analysis of the next section let us consider some examples. A first example is given by embedding $U(1)_{M}$ in $E_{7}$ according to the maximal-subgroup decomposition

$$
E_{7} \supset E_{6} \times U(1) .
$$

Using the branching rules 21]

$$
\begin{aligned}
& \mathbf{5 6} \rightarrow \mathbf{2 7 _ { 1 }}+\overline{\mathbf{2 7}}_{-1}+\mathbf{1}_{3}+\mathbf{1}_{-3}, \\
& \mathbf{1 3 3} \rightarrow \mathbf{7 8 _ { 0 }}+\mathbf{2 7 _ { - 2 }}+\overline{\mathbf{2 7}}_{2}+\mathbf{1}_{0},
\end{aligned}
$$

we see that $q_{\min }=1$ so that $n=$ integer. Discarding neutral fields, we see that the fermion representations under $E_{6} \times G_{2} \times U(1)_{M}$ which can give rise to fermion zero modes on $\mathbf{S}^{2}$ are,

$$
(\mathbf{2 7}, \mathbf{1 4})_{1}+(\overline{\mathbf{2 7}}, \mathbf{1 4})_{-1}+(\mathbf{1}, \mathbf{1 4})_{3}+(\mathbf{1}, \mathbf{1 4})_{-3},
$$

for the $E_{7}$ hyperinos and

$$
(\mathbf{2 7}, \mathbf{1 4})_{-2}+(\overline{\mathbf{2 7}}, \mathbf{1 4})_{2}
$$

for the $E_{7}$ gauginos.

As a second example, consider the successive maximalsubgroup decompositions

$$
E_{7} \supset S O(12) \times S U(2) \supset S O(10) \times S U(2) \times U(1),
$$

and identify the last $U(1)$ factor with $U(1)_{M}$. Using the branching rules

$$
\begin{aligned}
56 & \rightarrow(12,2)+(32,1), \\
133 & \rightarrow(1,3)+\left(32^{\prime}, 2\right)+(66,1),
\end{aligned}
$$

for $E_{7} \supset S O(12) \times S U(2)$ and

$$
\begin{aligned}
& \mathbf{1 2} \rightarrow \mathbf{1}_{1}+\mathbf{1}_{-1}+\mathbf{1 0}_{0}, \\
& \mathbf{3 2} \rightarrow \mathbf{1 6}_{1}+\overline{\mathbf{1 6}}_{-1}, \\
& \mathbf{3 2} \mathbf{2}^{\prime} \rightarrow \mathbf{1 6}_{-1}+\overline{\mathbf{1 6}}_{1}, \\
& \mathbf{6 6} \rightarrow \mathbf{1}_{0}+\mathbf{1 0}_{2}+\mathbf{1 0} \mathbf{0}_{-2}+\mathbf{4 5},
\end{aligned}
$$

for $S O(12) \supset S O(10) \times U(1)$, we see again that, $q_{\min }=1$ and $n=$ integer. Discarding neutral fields, we find that the muliplets of $S O(10) \times S U(2) \times G_{2} \times U(1)_{M}$ which can have fermion zero modes on $\mathbf{S}^{2}$ are

$$
\begin{aligned}
& (\mathbf{1}, \mathbf{2}, \mathbf{1 4})_{1}+(\mathbf{1}, \mathbf{2}, \mathbf{1 4})_{-1} \\
& +(\mathbf{1 6}, \mathbf{1}, \mathbf{1 4})_{1}+(\overline{\mathbf{1 6}}, \mathbf{1}, \mathbf{1 4})_{-1},
\end{aligned}
$$


for the hyperinos and

$$
\begin{aligned}
& (\mathbf{1 6}, \mathbf{2}, \mathbf{1 4})_{-1}+(\overline{\mathbf{1 6}}, \mathbf{2}, \mathbf{1 4})_{1} \\
& +(\mathbf{1 0}, \mathbf{1}, \mathbf{1 4})_{2}+(\mathbf{1 0}, \mathbf{1}, \mathbf{1 4})_{-2},
\end{aligned}
$$

for the $E_{7}$ gauginos. In the above examples, the representations (33) and (38) are understood as halfhypermultiplets.

\section{THE CHIRAL SPECTRUM AND (IN-)STABILITIES}

The monopole embeddings discussed in the previous section give rise to many chiral fermions in the complex representations of the unbroken gauge group in the effective $D=4$ theory. Using the formalism of [15] we can evaluate the content of the effective $D=4$ theory. Here we apply this formalism to the two examples of the previous section.

In the first example the unbroken gauge group is $E_{6} \times$ $G_{2} \times U(1)_{R} \times S U(2)_{K K}$, where $S U(2)_{K K}$ denotes the Kaluza-Klein gauge group originating from the isometries of $\mathbf{S}^{2}$. The chiral fermions originate from the $\mathbf{2 7}$ 's and the $\overline{\mathbf{2 7}}$ 's. We can regard all the $D=4$ fermions as lefthanded Weyl spinors. The chiral fermions originating from the decomposition of $\mathbf{5 6}$ of $E_{7}$ then are

$$
2(\overline{\mathbf{2 7}}, \mathbf{1 4}, \mathbf{n})_{0},
$$

while the fermions originating from the decomposition of the adjoint of $E_{7}$ produce

$$
(27,14,2 \mathrm{n})_{1}+(27,14,2 \mathrm{n})_{-1},
$$

with the subscripts here denoting the $U(1)_{R}$ charges.

In the second example the unbroken gauge group in $D=4$ is $G=S O(10) \times S U(2) \times G_{2} \times S U(2)_{K K} \times U(1)_{R}$. The spectrum of the $D=4$ chiral fermions is given by

$$
2(\overline{\mathbf{1 6}}, \mathbf{1}, \mathbf{1 4}, \mathbf{n})_{0},
$$

and

$$
(16,2,14, \mathbf{n})_{1}+(16,2,14, \mathbf{n})_{-1} .
$$

It is clearly seen that the spectrum in both cases is free from all chiral anomalies, because $E_{6}$ and $S O(10)$ are safe groups in $D=4$ and the $U(1)_{R}$ couplings are obviously vectorlike. It is also seen that there is no value of $n$ which produces a realistic spectrum. One can study other embeddings with the aim of reducing the gauge group and the number of families. For example, the group $G_{2}$ can be broken completely by the embedding of a monopole in an $S U(2)$ subgroup of $G_{2}$ relative to which the branching is $\mathbf{1 4}=\mathbf{3}+\mathbf{1 1}$. By itself this will produce only a vectorlike theory in $D=4$ with an unbroken group $E_{7} \times S U(2)_{K K} \times U(1)_{R}$. However, combined with other monopoles in the manner described above, one can break the group down to $S O(10) \times S U(2)_{K K}$. The number of families will nevertheless be still large.

Apart from the proliferation of the number of families and other shortcomings for a realistic model building with tree level considerations (such as the absence of a realistic Higgs spectrum and Yukawa couplings at tree level), there is a fundamental difficulty with all such compactifications. In fact it seems that, with the exception of the simplest compactifying solution in which the monopole is embedded in the $U(1)_{R}$ factor, all other compactifications are unstable. To see this, let us embed the momopole in one or both of the non-Abelian factors. Denote by $V$ one of the excitations of the vector potential tangent to $\mathbf{S}^{2}$ and charged with respect to $U(1)_{M}$. This vector has the components $V_{ \pm}$with respect to a complex basis in the tangent space of $\mathbf{S}^{2}$. We also have the reality condition $V=V^{\dagger}$. As a Lie-algebra-valued vector we can write $V$ as $V=U_{+}^{r} T_{r}+W_{+}^{r} T_{r}^{\dagger}$, where $U$ and $W$ are complex fields and the $T$ 's are among the charged generators of the gauge group. For example they can be the generators of $E_{7}$ in the directions of $\mathbf{1 0}_{2}$ or $\mathbf{1 6}_{1}$ of the previous section. In order to be able to write down a general formula which can be applied for any model of this kind, denote the $U(1)_{M}$ charge of $U$ or $W$ by $q$. The mass spectrum of $D=4$ spin-zero fields resulting from such a $D=6$ object is given by 22.

$$
a^{2} M^{2}=\ell(\ell+1)-(\lambda-1)^{2},
$$

where $\ell=|\lambda|,|\lambda|+1, \ldots$ and $\lambda=1+\frac{n}{2} q$. It is easy to see that for all those fields for which $n q \leq-2$ there is a tachyon. For example, with positive $n$, the leading mode in the spectrum of $\mathbf{1 0}_{2}$ will be a tachyon of squared mass $-n / 2 a^{2}$.

The only way to avoid this conclusion is to find an embedding for which $|n q|=1$ for all the excitations. With integer $n$ and $q$ we then need to have $n= \pm 1$ and $q= \pm 1$ for all the fields. Such an embedding is guaranteed to exist in all cases where there is a gauge group factor $G_{A}$ that has a maximal-subgroup decomposition $G_{\alpha} \supset H \times U(1)$ with $H$ simple and where all fermions charged under this group transform in the adjoint. This is exactly what happens in the $E_{7} \times E_{6} \times U(1)_{R}$ model, where the monopole can be embedded in the "hidden" $E_{6}$ that gives rise to $\mathbf{1 6}$ 's of $S O(10)$ with $q= \pm 1$. The monopole with the minimal charge of \pm 1 gives thus a stable compactification with two chiral families of $S O(10)$ in the 16-dimensional representation transforming as singlets under the unbroken $E_{7} \times S U(2)_{K K}$ but charged relative to the unbroken $U(1)_{R}$. It seems difficult to obtain an analogous result for the new model presented in this 
paper essentially because any embedding which produces integer $q$ 's necessarily contains fields for which $|q| \geq 2$. The only exception is of course the half-supersymmetric solution in which the monopole is embedded in $U(1)_{R}$. This embedding will leave $E_{7} \times G_{2}$ unbroken.

\section{CONCLUSIONS}

In this paper, we have demonstrated the existence of a second consistent $\mathcal{N}=1$ gauged supergravity model in six dimensions besides the old $E_{7} \times E_{6} \times U(1)_{R}$ model. The model found here is based on the $E_{7} \times G_{2} \times U(1)_{R}$ gauge group, with hypermatter transforming as a halfhypermultiplet in the pseudoreal representation $(\mathbf{5 6}, \mathbf{1 4})$. The theory satisfies a set of very stringent anomaly constraints and turns out to be free of local and global anomalies. Also, as its $E_{7} \times E_{6} \times U(1)_{R}$ sibling or, in fact, any other model of this type, the theory admits a monopole compactification on $\mathbb{R}^{4} \times \mathbf{S}^{2}$. Despite the fact that embedding monopole type configurations in $E_{7} \times G_{2}$ will produce many chiral fermions in $D=4$, it seems that all such solutions are perturbatively unstable. On the other hand, if the monopole is identified with the vector potential of $U(1)_{R}$ only, a stable compactification will be obtained and, with the choice of $n=1$, half of the $D=6$ supersymmetries will be unbroken. The model will clearly inherit all the brane solutions discovered so far in the context of $\mathcal{N}=1$ supergravity models in six dimensions, to some of which we referred in Section \

As far as the tachyons are concerned, one may adopt the point of view that they are welcome in the context of an effective theory as they are natural candidates for $D=4$ Higgs fields. The quartic term in the potential for such fields will come from the self-coupling of the $D=6$ gauge fields and their vev will break the $E_{6} \times S U(2)_{K K}$ $\left(\right.$ or $\left.S O(10) \times S U(2)_{K K}\right)$ at the KK scale. Such an origin for the Higgs fields has been considered before as a possible solution to the hierarchy problem. In order for this interpretation to be complete, one needs to look for new stable solutions of the six-dimensional field equations which would correspond to the minimum of the potential for the tachyons interpreted as Higgs fields. These solutions will necessarily break the spherical symmetry and their construction may give a geometrical origin to the Higgs mechanism. It will be interesting to find such solutions.

On the other hand, for phenomenological reasons, we may want to prevent certain modes from becoming tachyonic. In this paper we gave a necessary and sufficient condition for this to happen. Namely, in order for an excitation of an internal component of the gauge field not to be tachyonic, it is sufficient that $|n q|=1$, where $n q$ is understood as the sum of the individual $n q$ 's over all the monopole directions with respect to which the corre- sponding excitation is charged.

A final question, motivated by the uniqueness of the $D=6$ gauged supergravities under consideration and the fact that they cannot be constructed through straightforward string or M-theory compactifications, refers to their origin in terms of a higher-dimensional fundamental theory. Although previous experience might suggest that such models possibly arise due to some new mechanism involving non-perturbative physics, such a mechanism has not been identified up to date.

\section{APPENDIX A: ANOMALY POLYNOMIALS}

The anomaly structure of $D=6$ theories is encoded in a set of formal eight-forms, called anomaly polynomials. In our conventions, the gravitational anomaly polynomials [3] are given by

$$
\begin{aligned}
I_{8}^{1 / 2}(R) & =\frac{1}{360} \operatorname{tr} R^{4}+\frac{1}{288}\left(\operatorname{tr} R^{2}\right)^{2}, \\
I_{8}^{3 / 2}(R) & =\frac{49}{72} \operatorname{tr} R^{4}-\frac{43}{288}\left(\operatorname{tr} R^{2}\right)^{2}, \\
I_{8}^{A}(R) & =\frac{7}{90} \operatorname{tr} R^{4}-\frac{1}{36}\left(\operatorname{tr} R^{2}\right)^{2} .
\end{aligned}
$$

The gauge anomaly polynomials are given by

$$
\begin{aligned}
I_{8}^{1 / 2}(F) & =\frac{2}{3} \operatorname{tr} F^{4}, \\
I_{8}^{1 / 2}\left(F_{A}, F_{B}\right) & =4 \operatorname{tr} F_{A}^{2} \operatorname{tr} F_{B}^{2}, \\
I_{8}^{3 / 2}(F) & =\frac{10}{3} \operatorname{tr} F^{4},
\end{aligned}
$$

where the second polynomial applies to the case where product representations are present. Finally, the polynomials corresponding to mixed anomalies are

$$
\begin{aligned}
& I_{8}^{1 / 2}(F, R)=-\frac{1}{6} \operatorname{tr} R^{2} \operatorname{tr} F^{2}, \\
& I_{8}^{3 / 2}(F, R)=\frac{19}{6} \operatorname{tr} R^{2} \operatorname{tr} F^{2} .
\end{aligned}
$$

Here, the superscripts $1 / 2,3 / 2$ and $A$ refer to a spin $1 / 2$ fermion, a spin $3 / 2$ fermion and a $2-$ form potential respectively. The above anomaly polynomials correspond to Weyl spinors of positive chirality and 2-form potentials with self-dual field strengths. For negative-chirality spinors or anti-self-dual field strengths, the sign of the anomaly is reversed.

Acknowledgement. This work is supported by the EPEAEK programmes "Heraclitus" and "Pythagoras" and co-funded by the European Union (75\%) and the Hellenic State (25\%). 
[1] A. Salam and E. Sezgin, Phys. Lett. B 147, 47 (1984).

[2] G. W. Gibbons, R. Güven and C. N. Pope, Phys. Lett. B 595, 498 (2004) arXiv:hep-th/0307238.

[3] L. Alvarez-Gaumé and E. Witten, Nucl. Phys. B 234, 269 (1984).

[4] M. B. Green, J. H. Schwarz and P. C. West, Nucl. Phys. B 254, 327 (1985).

[5] J. Erler, J. Math. Phys. 35, 1819 (1994) arXiv:hep-th/9304104.

[6] E. Witten, Nucl. Phys. B 460, 541 (1996) arXiv:hep-th/9511030.

[7] J. H. Schwarz, Phys. Lett. B 371, 223 (1996) arXiv:hep-th/9512053.

[8] T. Gherghetta and A. Kehagias, Phys. Rev. Lett. 90 (2003) 101601 arXiv:hep-th/0211019; Phys. Rev. D 68, 065019 (2003) arXiv:hep-th/0212060.

[9] S. D. Avramis and A. Kehagias, Phys. Rev. D 71 (2005) 066005 arXiv:hep-th/0407221.

[10] S. Randjbar-Daemi, A. Salam, E. Sezgin and J. Strathdee, Phys. Lett. B 151 (1985) 351.

[11] H. Nishino and E. Sezgin, Nucl. Phys. B 278, 353 (1986).
[12] H. Nishino and E. Sezgin, Nucl. Phys. B 505, 497 (1997) arXiv:hep-th/9703075.

[13] R. Güven, J. T. Liu, C. N. Pope and E. Sezgin, Class. Quant. Grav. 21 (2004) 1001 arXiv:hep-th/0306201.

[14] Y. Aghababaie et al., JHEP 0309 (2003) 037 arXiv:hep-th/0308064.

[15] S. Randjbar-Daemi, A. Salam and J. Strathdee, Nucl. Phys. B 214 (1983) 491.

[16] S. Randjbar-Daemi and E. Sezgin, Nucl. Phys. B 692, 346 (2004) arXiv:hep-th/0402217.

[17] C. P. Burgess, F. Quevedo, G. Tasinato and I. Zavala, JHEP 0411 (2004) 069 arXiv:hep-th/0408109.

[18] E. Witten, Phys. Lett. B 117, 324 (1982).

[19] E. Bergshoeff, T. W. Kephart, A. Salam and E. Sezgin, Mod. Phys. Lett. A 1, 267 (1986).

[20] M. Bershadsky and C. Vafa, arXiv:hep-th/9703167

[21] R. Slansky, Phys. Rept. 79, 1 (1981).

[22] S. Randjbar-Daemi, A. Salam and J. Strathdee, Phys. Lett. B 124 (1983) 345 [Erratum-ibid. B 144 (1984) 455]. 